\title{
Denture Adhesives - Knowledge and Tradition among Denture Wearers in Western Maharashtrian Population - A Survey
}

\author{
Shivsagar Tewary ${ }^{1}$, Sweta Bajarang Shinde 2 , Karuna Gajanan Pawashe ${ }^{3}$, \\ Pronob Kumar Sanyal ${ }^{4}$, Shubha Joshi ${ }^{5}$, Uzma Belgaumi ${ }^{6}$
}

\begin{abstract}
${ }^{1}$ Department of Prosthodontics, School of Dental Sciences, Krishna Institute of Medical Sciences Deemed to be University, Karad, Maharashtra, India. ${ }^{2}$ Department of Prosthodontics, School of Dental Sciences, Krishna Institute of Medical Sciences Deemed to be University, Karad, Maharashtra, India. ${ }^{3}$ Department of Prosthodontics, School of Dental Sciences, Krishna Institute of Medical Sciences Deemed to be University, Karad, Maharashtra, India. ${ }^{4}$ Department of Prosthodontics, School of Dental Sciences, Krishna Institute of Medical Sciences Deemed to be University, Karad, Maharashtra, India. ${ }^{5}$ Department of Prosthodontics, School of Dental Sciences, Krishna Institute of Medical Sciences Deemed to be University, Karad, Maharashtra, India. ${ }^{6}$ School of Dental Sciences, Krishna Institute of Medical Sciences Deemed to be University, Karad, Maharashtra, India.
\end{abstract}

\section{ABSTRACT}

\section{BACKGROUND}

Denture adhesives play a vital role in retention of complete dentures, wherever indicated. Dentists need to know about denture adhesives to be able to identify those patients who actually need them and to be able to educate them about the advantages, disadvantages and correct use of these products. The aim of the study was to evaluate the knowledge about denture adhesives among complete denture wearers indicated for use of denture adhesives visiting Dental Colleges in Western Maharashtra.

\section{METHODS}

The study was conducted on 300 completely edentulous patients with conventional complete dentures indicated for use of denture adhesives.

\section{RESULTS}

Patients $(n=300)$ who were wearing ill - fitting complete dentures and required use of denture adhesives were selected, their response about knowledge was assessed by questionnaire survey and those who knew about it were instructed to demonstrate the application of denture adhesive to the intaglio surface of complete denture. $34.7 \%$ $\left(n_{1}=104\right)$ of the total subjects $(n=300)$ had heard about denture adhesives. Out of these 104 subjects, $69.2 \%\left(\mathrm{n}_{1}=72 ; \mathrm{CP}=24 \%\right)$ had used and $77.8 \%\left(\mathrm{n}_{1}=81 ; \mathrm{CP}=27 \%\right)$ knew when to use denture adhesives. Out of 72 subjects who had used denture adhesive, only $30.5 \%(\mathrm{CP}=7.3 \%)$ could demonstrate the adequate technique to use denture adhesive.

\section{CONCLUSIONS}

Knowledge about use of denture adhesives for those who are in need per se has to be spread along with right technique of its use. The indication of its use, and correct technique/techniques of application, should be taught to the patients who demand the use of denture adhesives.
Corresponding Author: Dr. Shivsagar Tewary, Associate Professor, Department of Prosthodontics, School of Dental Sciences, Krishna Institute of Medical Sciences, Deemed to be University, Karad, Maharashtra, India.

E-mail: ssagartewary@gmail.com

DOI: $10.14260 /$ jemds/2020/456

How to Cite This Article:

Tewary S, Shinde SB, Pawashe KG, et al. Denture adhesives- knowledge and tradition among denture wearers in Western Maharashtrian population- a survey. J. Evolution Med. Dent. Sci. 2020;9(30):2089-2092, $10.14260 /$ jemds $/ 2020 / 456$

Submission 11-03-2020,

Peer Review 18-06-2020

Acceptance 24-06-2020,

Published 27-07-2020.

Copyright (C) 2020 JEMDS. This is an open access article distributed under Creative Commons Attribution License [Attribution 4.0 International (CC BY 4.0)]

\section{KEY WORDS}

Adhesives, Questionnaire, Knowledge Tradition, Dentures 


\section{BACKGROUND}

Although the rate of edentulism has been falling over the past 30 years, there are still many countries with a large number of edentulous patients who are in need of treatment with complete dentures ${ }^{1}$. It is known that not all complete denture wearers are able to adapt to their dentures, even if they are well-fitting and well-made. Many complaints are reported by patients who use these prostheses, especially with mandibular dentures, including problems of retention and instability, chewing difficulties, and low confidence, quality of life and satisfaction. ${ }^{2,3}$ The ability to chew has been considered a determining factor for the satisfactory acceptance of dentures. ${ }^{4}$ Successful complete denture treatment combines exemplary technique, effective patient rapport and education and familiarity with all possible management options to provide the highest degree of patient satisfaction. ${ }^{5}$ Management of completely edentulous patients with conventional techniques has been considered as one of the most difficult treatment procedures. Denture adhesives have a legitimate place in prosthetic dental treatment. Denture adhesives benefit denture patients with improved fit, comfort, chewing ability and confidence if used properly. ${ }^{6-9}$

Use of adhesives in patients with compromised denture bearing areas adds to their confidence thereby increasing the ability to adapt to the new prosthesis. Even the best Dentists don't warrant a complete stability with highest degree of retention for conventional complete dentures owing to various factors including patient compliance. However, dentists are reluctant to prescribe them for fear that it indicates their failure to provide an adequate denture. ${ }^{7}$ Dentists need to identify those patients who actually need adhesives; be able to educate them about the advantages, disadvantages and correct use of these products. ${ }^{10}$ There is more or less restraining attitude of the profession towards denture adhesives but it was shown that a substantial proportion of denture wearers (7-33\%) had tried denture adhesives or were currently regular users. ${ }^{7}$

Some dental surgeons advise the use of an adhesive during the first weeks of wearing new dentures for some elderly patients, who experience the loss of their remaining teeth, in order to give the denture greater retention and to supply patient confidence. Denture adhesives are supplied as a paste, powder or cream. It may also be a helpful aid for the patients wearing an immediate denture to reduce discomfort. ${ }^{11}$ The use of a denture adhesive improves the retention of dentures by increasing the adhesive and cohesive properties, as well as the viscosity of the medium between the dentures and supporting tissues, eliminating voids in the space between them ${ }^{9}$. As the Adhesive powders absorb water, they swell to many times their original volume and the anions so formed, interact with cations in the proteins in the oral mucous membrane. The viscosity of the adhesive is increased by the thick saliva formed, thereby increasing the denture retention. The increased viscosity of the adhesive creams results in their lateral spread excluding air and saliva thereby increasing the retention. Thus, patients may use dental adhesives at home without understanding their effects, while dentists will not acknowledge denture adhesives without knowing their effects. ${ }^{12}$
We wanted to evaluate the knowledge regarding use and application of denture adhesives among denture wearers indicated for use of denture adhesives visiting the Dental Colleges in Western Maharashtra.

\section{METHODS}

The Institutional protocol committee and University ethical committee's approval was obtained for the questionnairebased study (survey). This was observational study conducted on patients visiting the OPD of Prosthodontic specialty; School of Dental Sciences; Krishna Institute of Medical Sciences; Deemed to Be University; Karad; Maharashtra. Inclusion criteria were completely edentulous patients who were wearing conventional complete dentures and their complete dentures were indicated for use of denture adhesives and willing to visit the clinic for denture adjustments as a recall patient. Patients whose complete dentures were indicated for relining/ rebasing procedures; have any systemic illnesses/any medically compromised condition that may make participating in the study difficult, are unable to understand and respond to the questionnaires used in the study, wearing metal-reinforced complete dentures, have maxillofacial defects covered by prosthetics, patients using tissue conditioners, and/or have severe oral dryness were excluded from the study. The sampling technique selected was convenient sampling. 329 patients were identified, among which 300 patients participated. The survey was conducted by instructing the patients to fill a questionnaire. The questionnaire was prepared and validated. Questionnaire copy is enclosed (table/Fig. 1). The questionnaire was translated in local language and translated back to English by bilingual expert. Content validity was assessed by a pilot study with close ended yes-no type of questions.

\section{Statistical Analysis}

The data obtained was subjected to statistical analysis using Statistical Package for Social Sciences Software and is used to evaluate the responses by frequency and percentage obtained.

\section{RESULTS}

Table/Fig 2 explains that out of 72 subjects who had used denture adhesive, only $30.5 \%(\mathrm{CP}=7.3 \%)$ could demonstrate the adequate technique to use denture adhesive. Though they could demonstrate the method to use denture adhesive, only $20 \%$ of them accomplished the method satisfactorily.

In the present study, all patients who were wearing ill fitting complete denture and required use of denture adhesives were assessed ( $n=300)$. Only $34.7 \%\left(n_{1}=104\right)$ of them had heard about denture adhesives. Out of these 104 subjects, $69.2 \%\left(\mathrm{n}_{1}=72 ; \mathrm{CP}=24 \%\right)$ had used and $77.8 \%\left(\mathrm{n}_{1}=\right.$ $81 ; \mathrm{CP}=27 \%$ ) knew when to use denture adhesives. However, only $15.3 \%\left(\mathrm{n}_{1}=16 ; \mathrm{CP}=5.3 \%\right)$ of these 104 subjects knew when not to use denture adhesive. Out of 72 subjects who had used denture adhesive, only $30.5 \%(\mathrm{CP}=7.3 \%)$ could demonstrate the adequate technique to use denture adhesive. Though they could demonstrate the method to use denture 
adhesive, only $20 \%$ of them accomplished the method satisfactorily.

\begin{tabular}{|c|c|c|c|}
\hline Survey Questionnaire??? & $\begin{array}{c}\text { Frequency } \\
\text { of Yes } \\
\left(\mathbf{n}_{1}\right)\end{array}$ & $\begin{array}{c}\text { Frequency } \\
\text { of No. (n) }\end{array}$ & $\begin{array}{c}\text { Total } \\
\left(\mathbf{n}_{1}+\mathbf{n}_{2}=\right. \\
\mathbf{n})\end{array}$ \\
\hline Do you wear complete denture? & 300 & $0 \mathrm{z}$ & 300 \\
Does your prosthesis fit properly? & 0 & 300 & 300 \\
Have you ever heard about denture adhesives? & 104 & 196 & 300 \\
Have you ever used denture adhesives? & 72 & 32 & 104 \\
Do you know when to use denture adhesives? & 81 & 23 & 104 \\
Do you know when not to use denture adhesive? & 16 & 88 & 104 \\
Can you demonstrate how to use denture & 22 & 50 & 72 \\
adhesive? & & \\
\hline
\end{tabular}

Table/Fig 1. Frequency of "Yes" ( $\left.n_{1}\right)$, Frequency of "No" $\left(n_{2}\right)$,

Total Subjects for the Question (n) for Each Question

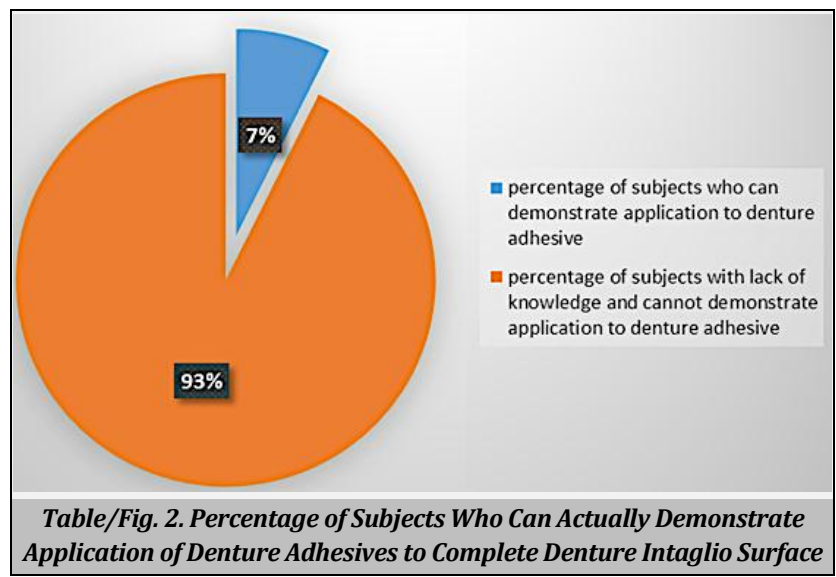

\section{DISCUSSION}

With an increase in life expectancy, there is a proportionate increase in edentulous patients. Though advent of advanced treatments like dental implants have been exercised by edentulous patients, the conventional removable complete dental prosthesis still remains one of the most preferred choice. Since patients approach for tertiary treatment care for their edentulous condition at very late stage, most of these conditions are associated with resorbed ridges, especially mandibular arches. Such resorbed ridges rehabilitated by conventional removal dental prosthesis pre-requisites use of denture adhesives. Denture adhesives are materials used to adhere the denture to oral mucosa. They fill gap caused by shrinking bone and gives temporary relief from the loosening dentures, thereby forming an important adjunct in providing retention to dentures. ${ }^{10}$ These are commercially available nontoxic, soluble materials that when applied to the tissue surface of the denture enhance their retention, stability and performance ${ }^{5}$ K. Shay has described the mechanism of action of adhesives. ${ }^{13}$

Many authors have reviewed use of denture adhesives. ${ }^{14-}$ 17 Jagger et al have outlined additional situations where the use of denture adhesives becomes mandatory for the improvement of the quality of retention. These are cases of severely atrophied edentulous ridges of severe grade; patients having lack of neuromuscular control (example: stroke and parkinsonism) which provides inadequate tissue support.10 Denture adhesives may also give psychological confidence for the patient as it supplements retention and stability especially during occasions of public interaction. ${ }^{18}$ Malhotra $\mathrm{P}$ et al carried out a study to compare the efficacy of different commercially available denture adhesives in relation to their retentive ability in patients with good well - formed mandibular ridges and in patients with resorbed and flat mandibular ridges. They concluded that all denture adhesives investigated in the study exhibited significantly higher retentive ability than saliva and significant improvement in retention was observed in patients with poor foundation as well good supporting tissues. ${ }^{19}$

Actually, the use of denture adhesives and their role in prosthodontics has been a conflicted topic, both in clinical practice and dental education. This conflicting viewpoint of dental professionals can be described as the traditional historical approach and the advocate approach. ${ }^{18}$ According to the traditional historical approach denture adhesives are not more than a poor substitute for proper fit, and the use of denture adhesives is related to poor clinical skills and a lack of prosthodontic practice. ${ }^{13-18}$ Dentists who support the advocate approach believe that when needed denture adhesives can facilitate clinical procedures of fabricating denture, patient satisfaction and acceptance. 18

An attempt to solve the dilemma regarding the use of denture adhesives among the academic prosthodontics and it was concluded that denture adhesives are useful adjunct in denture prosthesis service. Prosthodontists agreed with $100 \%$ consensus that denture adhesives were useful to provide additional retention and stability for patients who have inadequate oral anatomy. Education for both dentists and the patients is imperative for the proper use and avoid misuse of the same. ${ }^{18}$ Grounder $\mathrm{R}$ and Sathasivam $\mathrm{P}$ reviewed the importance of denture adhesives in complete denture patients and concluded that with proper use, denture adhesives are beneficial to the patient in increasing retention and stability, enhanced comfort, improved function, in providing psychological satisfaction, thus improving the edentulous patients quality of life (QOL). ${ }^{15}$

Ozan et al supported the concept that denture wearers are older and often have their present dentures for 10 years or more. In their survey, Seventy-three percent of the patients did not need a denture adhesive as they managed their dentures well. Eighty-seven per cent of the patients did not know that denture adhesives existed; eight percent of the patients had tried denture adhesive but none of them continued it on a regular basis. They suggested that the knowledge on denture adhesives is less and it should be taught more intensively at dental schools and be involved in continuing education programmes for general practitioners. ${ }^{21}$

Though most of the research claims denture adhesive to be harmless, there have been studies indicative of statistically significant degree of cytotoxicity in denture adhesives. The degree of toxicity was more in Fixon powder followed by Dentiro powder. Metrodent powder showed the least toxicity of all the powder forms of denture adhesives tested, while the least was evident in Fixon cream. ${ }^{20}$ The underlying causes of denture stomatitis may be related to the long-term use of denture adhesive, which may predispose individuals to oral candidiasis. Bates AM et al suggested that denture adhesive may inactivate innate immune mediators in the oral cavity increasing the risk of Candida albicans infections, but inclusion of anti-fungal antibiotics to denture adhesive may aid in prevention and treatment of Candida infections and denture stomatitis. ${ }^{22}$ More importantly, antifungal antibiotics retained 
their anti-Candida albicans activity in denture adhesive strongly suggesting that anti-fungal antibiotics could be candidates for inclusion in adhesive formulations and used as prescribed topical treatments for individuals in denture stomatitis ${ }^{23}$. Slaughter et al. stated that denture adhesives could contribute to the development of denture stomatitis, candidiasis, alveolar bone resorption and imbalance in oral flora, but not leukoplakia or oral cancer. ${ }^{18}$

Denture adhesives occupy a paramount role in complete denture rehabilitation, the current denture literature supports the use of denture adhesives and dispels some of the negative opinion that originate with them. ${ }^{19}$ However, the patient must be educated in the appropriate use of the material so that they enhance patient need and comfort.

It is important that dentists inform patients of the advantages and disadvantages of denture adhesives, their use as per manufacturers' instructions following specific guidelines for application and removal to prevent potential misuse. Furthermore, instructions and demonstrations clinically how to apply and remove adhesives correctly, and education of patients about the significance of routine recall appointments for removable prostheses must be done. They should not be used as an aid to compensate for denture deficiencies even though adhesives enhance denture performance. Patients should not use denture adhesives inadvertently without proper guidance and instructions from the dentists. ${ }^{17}$ The inference of this study could be attributed to inadequate knowledge about denture adhesive for those patients who are in need of it.

\section{CONCLUSIONS}

Knowledge about use of denture adhesives for those who are in need per se has to be spread along with the right technique of its use. The indication of its use correct technique/techniques of application should be taught to the patients who demand the use of denture adhesives. Detailed demonstrations, and visual aids should be provided. Also, its practical indications, and contra-indications, should be included in the teaching curriculum so that dentists emphasize the importance of denture adhesives in patients who demand it. Sound knowledge and experience of the dentist in treating complete denture patients is of crucial importance.

Financial or Other Competing Interests: None.

\section{REFERENCES}

[1] Carlsson GE, Omar R. The future of complete dentures in oral rehabilitation. A critical review. J Oral Rehabil 2010;37(2):143-56.

[2] Siadat H, Alikhasi M, Mirfazaelian A, et al. Patient satisfaction with implant-retained mandibular overdentures: a retrospective study. Clin Implant Dent Relat Res 2008;10(2):93-8.

[3] Emami E, Heydecke G, Rompre PH, et al. Impact of implant support for mandibular dentures on satisfaction, oral and general health-related quality of life: a meta-analysis of randomized Controlled trials. Clin Oral Implants Res 2009;20(6):533-44.
[4] De Souza e Silva ME, de Magalhaes CS, Ferreira e Ferreira E. Complete removable prostheses: from expectation to (dis) satisfaction. Gerodontology 2009;26(2):143-9.

[5] Zarb GA, Carlsson GE, Bolender CL. Boucher's Prosthodontic treatment for edentulous patient. $11^{\text {th }}$ edn. St.Louis: Mosby 1997.

[6] Adisman IK. The use of denture adhesives as an aid to denture treatment. J Prosthet Dent 1989;62(6):711-5.

[7] Coates AJ. Usage of denture adhesives. J Dent 2000;28(2):137-40.

[8] Coates AJ. Denture adhesives: a review. Aust Prosthodont J 1995;9:27-31.

[9] Grasso JE. Denture adhesive: changing attitudes. J Am Dent Assoc 1996;127(1):90-6.

[10] Jagger DC, Harrison A. Denture fixatives-an update for general dental practice. Br Dent J 1996;180(8):311-3.

[11] Stafford GD. Denture adhesives--a review of their uses and compositions. Dent Pract Dent Rec 1970;21(1):17-9.

[12] Kimoto S, Kawai Y, Gunji A, et al. Study protocol for a multi-center, randomized controlled trial to develop Japanese denture adhesive guidelines for patients with complete dentures: the Denture Adhesive Guideline trial: study protocol for a randomized controlled trial. Trials 2016;17(1):506.

[13] Shay K. Denture adhesives. Choosing the right powders and pastes. J Am Dent Assoc 1991;122(1):70-6.

[14] Musani S, Dugal R, Kothavade M. A review of denture adhesives used in the dental profession. Annals and Essences of Dentistry 2010;2(3):129-33.

[15] Gounder R, Sathasivam P. Importance of denture adhesive in complete denture patients - a review. International Journal of Recent Advances in Multidisciplinary Research 2016;3(6):1535-7.

[16] Papadiochou S, Emmanouil I, Papadiochou I. Denture adhesives: a systemic review. J Prosthet Dent 2015;113(5):391-7.

[17] Kumar PR, Shajahan PA, Mathews J, et al. Denture adhesives in prosthodontics: an overview. J Int Oral Health 2015;7(Suppl 1):93-5.

[18] Slaughter A, Katz RV, Grasso JE. Professional attitudes toward denture adhesives: a Delphi technique survey of academic prosthodontists. J Prosthet Dent 1999;82(1):80-9.

[19] Malhotra P, Yadav B, Yadav H, et al. A clinical study to assess the efficacy of commonly available denture adhesive materials in mandibular dentures. Int J Prosthodont Restor Dent 2013;3(4):125-30.

[20] Agarwal SK, Praveen G, Gupta S, et al. In vitro evaluation of cytotoxicity of denture adhesives. Indian J Dent Res 2011;22(4):526-9.

[21] Ozcan M, Kulak Y, Arikan A, et al. The attitude of complete denture wearers towards denture adhesives in Istanbul. J Oral Rehabil 2004;31(2):131-4.

[22] Bates AM, Garaicoa JL, Fischer CL, et al. Diminished antimicrobial peptide and antifungal antibiotic activities against Candida albicans in denture adhesive. Antibiotics (Basel) 2017;6(6):6

[23] Garaicoa JL, Fischer CL, Bates AM, et al. Promise of combining anti-fungal agents in denture adhesives to fight Candida species infections. J Prosthodont 2016;27(8):755-62. 